\title{
Abnormal Cancellous Bone Collagen Metabolism in Osteoarthritis
}

\author{
Jason P. Mansell and Allen J. Bailey
}

Collagen Research Group, Division of Molecular and Cellular Biology, University of Bristol, Langford, Bristol, United Kingdom

$B S 187 D U$

\begin{abstract}
Biochemical investigations into the pathogenesis of osteoarthritis have, for the last two decades, concentrated on the mechanisms involved in the destruction of the articular cartilage. Although bone changes are known to occur, the biochemistry of the collagenous matrix within osteoarthritic bone has received scant attention. We report that bone collagen metabolism is increased within osteoarthritic femoral heads, with the greatest changes occurring within the subchondral zone. Collagen synthesis and its potential to mineralize were determined by the carboxy-terminal propeptide content and alkaline phosphatase activity, respectively. These data supported elevated new matrix formation. Our finding of a three- to fourfold increase in TGF- $\beta$ in osteoarthritic bone indicates that this might represent a stimulus for the increased collagen synthesis observed. Of additional significance is the hypomineralization of deposited collagen in the subchondral zone of osteoarthritic femoral heads, supporting a greater proportion of osteoid in the diseased tissue. The cross-linking of collagen was similar to that observed for controls. In addition, the degradative potential of osteoarthritic bone was considerably higher as demonstrated by increased matrix metalloproteinase 2 activity, and again the greater activity was associated with the subchondral bone tissue.

The polarization exhibited in the metabolism of bone collagen from osteoarthritic hips might exacerbate the processes involved in joint deterioration by altering joint morphology. This in turn may alter the distribution of mechanical forces to the various tissues, to which bone is a sensitive responder. Bone collagen metabolism is clearly an important factor in the pathogenesis of osteoarthritis and certainly warrants further biochemical study. (J. Clin. Invest. 1998. 101:1596-1603.) Key words: osteoarthritis • bone • collagen • gelatinase $\bullet$ procollagen
\end{abstract}

\section{Introduction}

Osteoarthritis $(\mathrm{OA})^{1}$ represents a major cause of disability, particularly among the aging population; indeed, OA is the

\footnotetext{
Address correspondence to Jason P. Mansell, Collagen Research Group, Division of Molecular and Cellular Biology, University of Bristol, Langford, Bristol, UK BS18 7DU. Phone: 44-117-928-4779; FAX: 44-117-929-9898; E-mail: j.p.mansell@bris.ac.uk

Received for publication 9 June 1997 and accepted in revised form 4 February 1998
}

J. Clin. Invest.

(C) The American Society for Clinical Investigation, Inc. 0021-9738/98/04/1596/08 \$2.00

Volume 101, Number 8, April 1998, 1596-1603

http://www.jci.org most common form of joint disease (1). Despite much research into this condition, the etiology of OA is unknown, but it is generally agreed to be multifactorial. OA is invariably described as a loss of articular cartilage, with joint space narrowing accompanied by secondary bone changes. Consequently, the main thrust of research into OA has focused on the cartilage, the loss of which is believed by many to represent the initial event responsible for joint destruction, pain, and disability. Therefore, biochemical analysis of the underlying bone tissue has received scant attention despite the well known changes that occur, such as the formation of osteophytes and subchondral cysts, which are striking characteristics of the disease (2). Further, early histological analyses (3-5) provided some indication of unusual bone remodeling in OA, in particular that matrix deposition exceeded mineralization. Changes within the subchondral bone have been reported by Radin and his colleagues $(6,7)$, who postulated that a thickening of the underlying bone was a consequence of healing microfractures, which in turn resulted in disease development, but direct biochemical evidence was not available. Renewed interest regarding the importance of bone tissue in $\mathrm{OA}$ has come from the technetium uptake studies of Dieppe et al. (8), the increased bone uptake of technetium-labeled bisphosphonate correlating with disease severity in terms of joint space narrowing. Although of value in describing tissue changes in OA, these scintigraphic analyses extend only to the inorganic phase of bone; what peculiarities there might be to the collagenous scaffold into which the mineral is impregnated remain unknown.

The major component of joint tissues is bone, the metabolism and composition of which are fundamental to the biomechanical competence and overall health of the joint. Fibrous type I collagen is the major structural component of bone, accounting for $\sim 90 \%$ of the organic matrix. The function of the small amount of type $\mathrm{V}$ collagen $(\sim 3 \%)$ is uncertain, but based on the retention of its amino-terminal propeptide, it may be involved in regulation of type I collagen fiber size (9). In addition to its important supportive role, collagen provides a means of cellular communication, notably to the osteoblasts and osteocytes, a main function of which is believed to be the transduction of mechanical stimuli, which has important consequences for the regulation of bone mass (10-12).

The remodeling of bone tissue, whatever the stimulus, involves the synthesis and degradation of the collagenous framework. Our preliminary studies (13) demonstrated increased matrix metalloprotease (MMP) activity, but whether this is correlated with increased collagen synthesis or whether turn-

1. Abbreviations used in this paper: AP, alkaline phosphatase; DC, distal compartment; DHLNL, dihydroxylysinonorleucine; HP, hydroxylysyl pyridinoline; LHNL, lysinohydroxynorleucine; MMP, matrix metalloprotease; MT1-MMP, membrane-associated MMP; OA, osteoarthritis; PC, proximal compartment; PICP, carboxy-terminal propeptide of type I collagen; TIMP, tissue inhibitor of MMPs. 
over is uniform within the femoral head was not determined. Coordination of skeletal tissue turnover is fundamental to maintaining joint morphology, as highlighted, for example, in the "patchy" deformity associated with Paget's disease (14).

In this study, we have examined the difference in collagen synthesis between OA and nonarthritic controls by analysis of tissue alkaline phosphatase (AP) and the carboxy-terminal propeptide of type I collagen (PICP). The content of calcium in relation to the existing collagenous matrix was also determined, to compare the extent of tissue mineralization between control and OA cancellous bone tissue. Additional compositional studies focused on the cross-linking of collagen, namely the pyridinium and keto-imine cross-linking amino acids. Finally, the potential of bone tissue to degrade the collagen by members of the MMPs also formed part of the investigation. We have concentrated our efforts on gelatinase A, the most widely distributed MMP, which was reported recently to be capable of cleaving native fibrillar collagens (15). Furthermore, its activation is mediated by a specific mechanism (16), namely by the actions of a membrane-associated MMP (MT1-MMP), providing for selective potentiation independent of other MMPs. Furthermore, we have previously reported increased levels of pro and active gelatinase $\mathrm{A}$ in OA cancellous bone (13), an enzyme also found to be regulated by parathormone and estrogen in rat bone in vivo (17), and therefore considered to be important in bone collagen turnover.

\section{Methods}

\section{Source of hip joints}

12 OA femoral heads were kindly provided by Dr. Carole Westacott (Rheumatology Unit, University of Bristol). These specimens were collected from elderly (65-90 yr) females immediately after hip arthroplasty at the Bristol Royal Infirmary. Seven normal femoral heads were obtained at postmortem. These normal specimens were taken from cadavers within $48 \mathrm{~h}$ after death and stored at $4^{\circ} \mathrm{C}$. After collection, both $\mathrm{OA}$ and control specimens were stored frozen at $-20^{\circ} \mathrm{C}$, and analyses were completed within 2 mo of surgery.

\section{Tissue preparation}

Using a fine band saw, all femoral heads were sliced through the center to obtain a 2-3-mm section of tissue. Two discs of cancellous bone were isolated using a punch from each slice. The relationship of the sampled sites to the femoral head is presented in Fig. 1. A site immediately below $(1 \mathrm{~cm})$ and central to the insertion site of the Ligamentum teres is referred to as the proximal compartment (PC), and the second sample site, the distal compartment (DC), was taken near the existing head/neck junction. Unless stated otherwise, a total of seven PC and seven DC sites were taken from the normal femoral heads, and a total of twelve PC and twelve DC sites were taken for the OA specimens. All sampled sites were snap-frozen in liquid nitrogen and milled to a powder using a stainless steel mill which had been precooled using liquid nitrogen. Approximately $150 \mathrm{mg}$ of powdered bone was dispensed into polypropylene tubes $(6 \mathrm{ml}$; Sarstedt Ltd., Leicester, UK) and extracted with $1.8 \mathrm{ml}$ of $10 \mathrm{mM}$ triethanolamine (Sigma Chemical Co., Poole, Dorset, UK), pH 7.5, as described previously (13). Specimens were mixed thoroughly using micro stirring bars $(5 \times$ $3 \mathrm{~mm}$; Jencons [Scientific] Ltd., Leighton Buzzard, UK) over a magnetic stirring plate. After stirring the samples at $4^{\circ} \mathrm{C}$ for $1.5 \mathrm{~h}$, samples were centrifuged for $10 \mathrm{~min}$ at $10,000 \mathrm{rpm}$. An aliquot $(1.3 \mathrm{ml})$ of the extract was used for MMP determinations, and the remainder was divided for analysis of total protein, AP, and PICP. The insoluble pellet was analyzed for collagen content, calcium content, collagen crosslinking amino acids, and TGF- $\beta$.

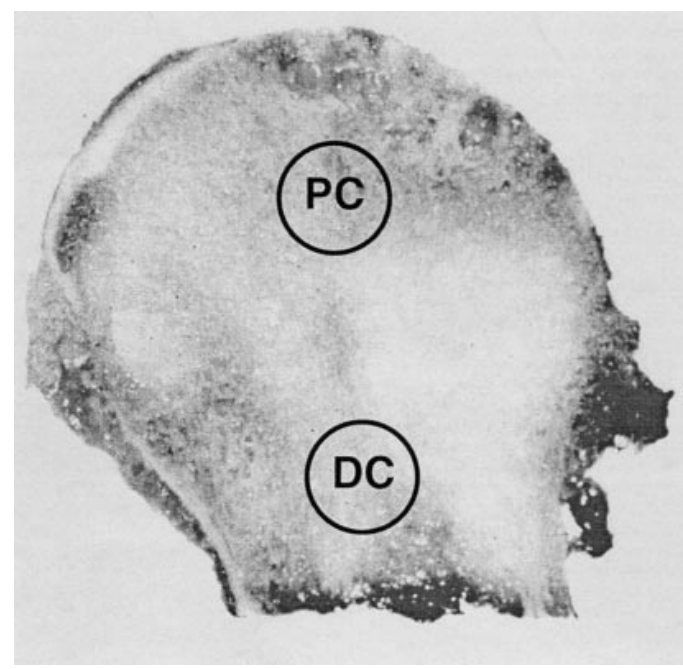

Figure 1. A slice from a human $\mathrm{OA}$ femoral head depicting the relationship of the two cancellous bone sample sites isolated for biochemical analysis. Each femoral head was sliced using a band saw in the plane of the long axis of the diaphysis. Each sample was isolated using a circular punch from a 2-3-mm-thick section of each femoral head.

\section{Soluble tissue components}

PICP. The PICP content of tissue supernatants $(100 \mu l$ of the original tissue extract per analysis) was quantified using the Prolagen- $\mathrm{C}^{\circledR}$ ELISA format (Metra Biosystems, Inc., Oxford, UK) according to the manufacturer's instructions. The Prolagen- $\mathrm{C}^{\circledR}$ assay is a sandwich immunoassay in a microtiter plate format using an anti-PICP mAb coated to the plate. A rabbit anti-PICP antiserum, a goat anti-rabbit AP conjugate, and a $p$-nitrophenol phosphate substrate allow for quantification of the propeptide in tissue extracts. Developed plates were read at $405 \mathrm{~nm}$ using a microtiter plate reader (Multiskan MCC/ 340; Labsystems, Helsinki, Finland). The presence of PICP in the extracellular space stoichiometrically reflects type I collagen synthesis (18). PICP was readily extracted from tissue samples, and using the Prolagen- $C^{\circledR}$ assay, it was found that $\sim 85-90 \%$ was recovered in the extraction procedure adopted on a separate set of samples. After the initial extraction, supernatants were harvested as described earlier, and the remaining matrix was resuspended in $1.8 \mathrm{ml}$ of fresh $10 \mathrm{mM}$ triethanolamine buffer. The extraction procedure was repeated an additional two times. Aliquots from each supernatant were assayed for PICP concentration, and from the data collected, an estimate of the extraction efficiency could be calculated. Further, duplicate specimens gave comparable extraction data; the observed differences between duplicate samples were $<10 \%$. Tissue PICP is expressed in relation to extracted protein (nanograms of PICP per milligram of protein).

AP. Analysis of tissue AP, a useful index of matrix formation and mineralization (19), was identical to that detailed previously (13). Briefly, undiluted aliquots were assayed for the enzyme using a specific autoanalyzer (Kone Instruments Corp., Espoo, Finland) with $p$-nitrophenol phosphate as substrate. The levels of the enzyme are expressed in relation to the extracted protein (units of activity per milligram of protein).

Protein quantification. Protein in undiluted tissue extracts was analyzed on a Cobas Bio system (F. Hoffmann-La Roche, Ltd., Welwyn Garden City, UK) and determined using Bradford reagent (610-2; Sigma Chemical Co.). The protein standards used in the assay ranged between 15 and $50 \mathrm{mg} / \mathrm{dl}$ (610-11; Sigma Chemical Co.).

MMPs. Aliquots $(1.3 \mathrm{ml})$ from each preparation were dispensed 
into preweighed microcentrifuge tubes, frozen, and lyophilized. After drying, these samples were reconstituted appropriately and prepared for gelatin gel electrophoresis to identify pro and active gelatinase A (MMP-2). Briefly, freeze-dried samples were dissolved in $130 \mu \mathrm{l}$ of buffer comprised of $500 \mathrm{mM} \mathrm{NaCl}, 50 \mathrm{mM} \mathrm{CaCl}_{2}, 50 \mathrm{mM}$ Tris base, $\mathrm{pH}$ 7.5. An aliquot of the solubilized extract was then diluted 10 times in sample buffer as described by Laemmli (20) but containing twice the concentration of SDS ( $2 \% \mathrm{wt} / \mathrm{vol})$ and no reducing agent. The remainder of the sample was stored frozen at $-20^{\circ} \mathrm{C}$. Samples were electrophoresed on the day of their reconstitution in a $10 \%$ polyacrylamide (Bio-Rad Laboratories, Hemel Hempstead, UK) gel copolymerized with $0.75 \mathrm{mg} / \mathrm{ml}$ (final concentration) of gelatin (bovine skin; Sigma Chemical Co.). All zymography was performed using 1-mmthick minigels ( $4.9 \mathrm{ml}$ per resolving gel) prepared using mini PII electrophoresis apparatus (Bio-Rad Laboratories). Sample volumes for electrophoresis were calculated from the original tissue mass as described previously (13). Sample volumes for electrophoresis were inversely related to the initial tissue mass (according to the calculation $150 \mathrm{mg}$ /original mass $[\mathrm{mg}] \times 5 \mu \mathrm{l}$ ). After electrophoresis, gels were processed for MMP detection by the method described by Mansell et al. (17). Briefly, gels were rinsed three times in $2.5 \%$ (vol/vol) Triton X-100 (Sigma Chemical Co.) over a period of $15 \mathrm{~min}$, washed three times in distilled water, and then bathed in $50 \mathrm{ml}$ of $50 \mathrm{~mm} \mathrm{CaCl}_{2}, 50 \mathrm{mM}$ Tris base, $500 \mathrm{mM} \mathrm{NaCl}, \mathrm{pH} 7.8$, supplemented with aminophenylmercuric acetate (APMA; Sigma Chemical Co.) at a final concentration of $200 \mu \mathrm{mol} / \mathrm{liter}$. Gels were left to incubate in this mixture for $16 \mathrm{~h}$ at $37^{\circ} \mathrm{C}$. After this incubation period, gels were rinsed three times in distilled water and subsequently stained and destained as for conventional SDS-PAGE (20). The resulting zymograms were processed and scanned to quantitate the proteolytically clarified zones as detailed previously (17). Briefly, developed gels were scanned using a studioscan II (Agfa Division, Bayer Corp., Ridgfield Park, NJ) in transmittance mode with Fotolook SA 2.05 software operated from a Power Macintosh 7100. The images were analyzed in NIH image 1.55 in which absorbances are plotted as a response profile, allowing quantitation of the proteolytically clarified zones according to their areas and intensities. The correction adopted involved dividing the area obtained for the tissue extract by the area obtained for the loaded standard. In this way, plots of different gels could be compared when identical standards were electrophoresed. Confirmation that the observed zones were attributable to MMP activity was achieved by incubating identical gels, before development, in buffers containing either $5 \mathrm{mM}$ EDTA or $10 \mathrm{mM} \mathrm{1,10}$ phenanthroline (Sigma Chemical Co.). In each case, no proteolytically clarified zones appeared. Approximately $1.5 \mathrm{ng}$ of a human MMP-2 standard (Biogenesis Laboratories, Bournemouth, UK) was electrophoresed alongside tissue extracts to assist in the identification and quantification of the relevant zones.

\section{Insoluble tissue analyses}

Cross-linking amino acids. The remaining matrix was resuspended in $1 \mathrm{ml}$ PBS (prepared from tablets; Sigma Chemical Co.), pH 7.4, and treated with potassium borohydride (Sigma Chemical Co.) for $1 \mathrm{~h}$ to reduce the precursor keto-imine cross-linking amino acids to lysinohydroxynorleucine (LHNL) and dihydroxylysinonorleucine (DHLNL) as described previously (21). After reduction, concentrated $\mathrm{HCl}$ was added to a final $6 \mathrm{M}$ acid concentration. Samples were subsequently hydrolyzed in their original polypropylene tubes for $24 \mathrm{~h}$ at $110^{\circ} \mathrm{C}$, and the acid was then removed by freeze-drying. Dried samples were reconstituted into $500 \mu \mathrm{l}$ of distilled water, the bulk of which ( $90 \%$ ) was reserved for CF1 cellulose (Whatman International Ltd., Kent, UK) chromatography to concentrate the cross-linking amino acids, the remainder $(50 \mu \mathrm{l})$ for calcium and hydroxyproline determinations. CF1 cellulose prefractionation to enrich both reduced DHLNL and LHNL and mature pyridinium compounds (lysyl and hydroxylysylpyridinoline [HP]) and their subsequent quantitation by amino acid analysis (Alpha plus amino acid analyzer; Pharmacia Biotech, Loughborough, UK) was identical to that described previously (21). The location of the cross-links on chromatograms has been confirmed previ- ously with samples of authentic residues prepared in our laboratory. The cross-linking content of tissue samples is expressed in moles per mole of collagen.

Calcium content. Aliquots $(10 \mu \mathrm{l})$ were analyzed for calcium on a specific autoanalyzer (Kone Instruments Corp.) adopting the Raichem $^{\circledR}$ colorimetric assay (Hemagen Diagnostics, Inc., San Diego, CA). Bone collagen calcification is expressed as millimoles of calcium per mole of collagen from the original native tissue samples.

Collagen content. The collagen content of acid-hydrolyzed bone samples $(10 \mu \mathrm{l})$ was determined by hydroxyproline analysis (22) using a continuous flow autoanalyzer (ChemLab Instruments Ltd., Hornchurch, Essex, UK). Collagen content is expressed as micrograms of collagen per milligram of the original native sample mass.

$T G F-\beta$. Five normal and five OA bone samples isolated from the PC were prepared for analysis of TGF- $\beta$. The extraction of this cytokine from bone using $4 \mathrm{M}$ guanidine chloride $/ 260 \mathrm{mM}$ tetrasodium EDTA/30 mM Tris, $\mathrm{pH} 7.4$, was identical to that described by Dequeker and colleagues (23) except that acetone was used to defat previously water-washed powdered specimens. Bone samples were subjected to three acetone washes $(10 \mathrm{mg} / \mathrm{ml})$, each lasting $10 \mathrm{~min}$, by swirling at room temperature. The only other modification was substituting PMSF for the more stable and water soluble pefabloc (Boehringer Mannheim UK, Sussex, UK) as the serine protease inhibitor during the extraction procedure. Quantitation of TGF- $\beta$ was by ELISA (Genzyme Corp., Cambridge, MA). Lyophilized tissue extracts were reconstituted at a concentration of $2.5 \mathrm{mg} / \mathrm{ml}$ in $40 \mathrm{mM}$ $\mathrm{HCl}$ before incubation. This step, which took place at $4^{\circ} \mathrm{C}$ for $1 \mathrm{~h}$, results in the activation of TGF- $\beta$. Samples were subsequently neutralized and diluted, as instructed by the manufacturer, before dispensing onto the plate. Developed plates were read at $450 \mathrm{~nm}$ using a Multiskan MCC/340 microtiter plate reader. The tissue content of TGF- $\beta$ is expressed as nanograms of cytokine per milligram of dry, fat-free bone powder.

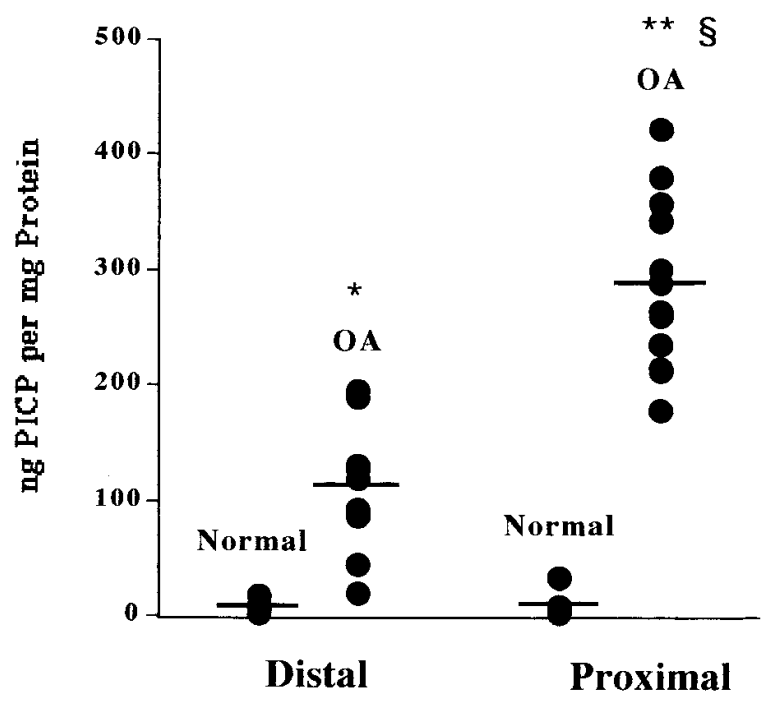

Figure 2. The tissue content of the PICP accurately reflects the stoichiometric synthesis of type I collagen. The data presented demonstrate highly significant differences in the synthetic ability of OA tissue samples compared with normal specimens. Both distal $(*)$ and proximal $(* *)$ compartments of OA tissue contained significantly $(P<0.0001)$ more PICP compared with normal samples. Of further significance is the degree of polarization of PICP within OA tissue preparations, with the proximal pole containing far greater quantities $\left({ }^{\S} P<0.0001\right)$ than the distal site. 


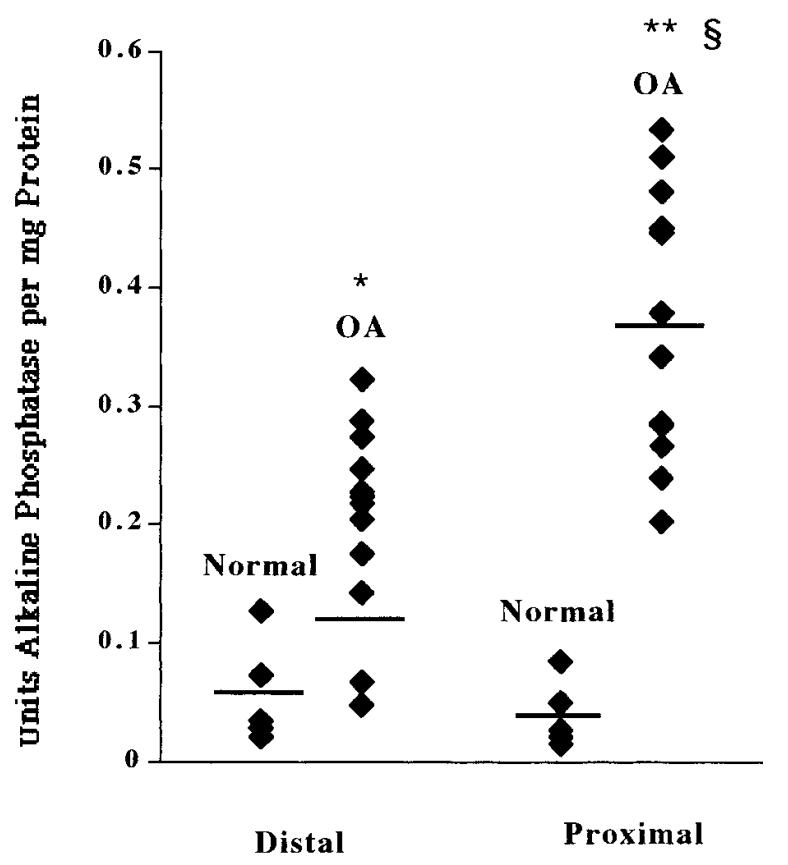

Figure 3. Comparison of the tissue content of AP at different bone sites. The enzyme is a useful indicator of bone matrix formation and mineralization potential. Significantly greater enzyme levels were recovered from both the distal $(* P<0.0005)$ and proximal $(* * P<$ 0.0001 ) regions of OA bone compared with site-matched normal samples. Note the clear polarization exhibited in the levels of AP between DC and PC for the OA tissue samples $\left({ }^{\S} P<0.0001\right)$.

\section{Statistical analysis}

Statistical analysis was performed using Minitab Statistical Software, version 10 . The Ryan-Joiner test was applied to all data to determine whether data were normally distributed. The subsequent test applied was a Student's $t$ test, without assuming equal variance. Paired analyses were performed for comparison between $\mathrm{PC}$ and DC for the same femoral head samples, unpaired analyses for comparisons between normal and diseased tissues.

Data obtained for LHNL and HP were found not to be normally distributed. The Mann Whitney U test was applied to these data. Differences between groups were assumed to be statistically significant where $P<0.05$. Unless stated otherwise, data are expressed as the mean \pm SEM.

\section{Results}

\section{Soluble extract analyses}

Synthesis of bone, type I collagen. Tissue supernatants were analyzed for PICP to determine the difference in type I collagen synthesis between normal and diseased tissue. The extent of type I collagen synthesis was greatest in the OA specimens (Fig. 2). The PC for OA contained significantly more $(P<$ $0.0001)$ PICP than site-matched normal samples $(288 \pm 22 \mathrm{ng}$ per $\mathrm{mg}$ protein vs. $11 \pm 6)$. The $\mathrm{DC}$ of $\mathrm{OA}$ also contained significantly $(P<0.0001)$ greater quantities of the propeptide compared with the normal samples $(112 \pm 15$ vs. $8 \pm 3)$. Of particular note is the significant $(P<0.0001)$ degree of polarity in the content of PICP between the PC and DC for OA-sampled sites, the former exhibiting the greater value. No such difference was observed for control samples.

Tissue extract analysis of AP. AP levels were greatest in the OA tissue samples studied (Fig. 3). The PC for OA contained $0.37 \pm 0.03 \mathrm{U}$ AP per $\mathrm{mg}$ of protein vs. $0.04 \pm 0.01(P<$ $0.0001)$ for site-matched controls. The DC for OA also contained significantly $(P<0.0005)$ more AP than site-matched normal specimens $(0.2 \pm 0.02$ vs. $0.06 \pm 0.02)$. OA femoral heads exhibited a clear polarization $(P<0.0001)$ in AP expression, with the greater levels found in the PC.

Collagenolytic potential of bone tissue. Both pro and active MMP-2 were elevated significantly in OA compared with normal tissue; indeed, very little of either enzyme species, from either site, was present in normal tissue, as is evident from the zymogram (Fig. 4).

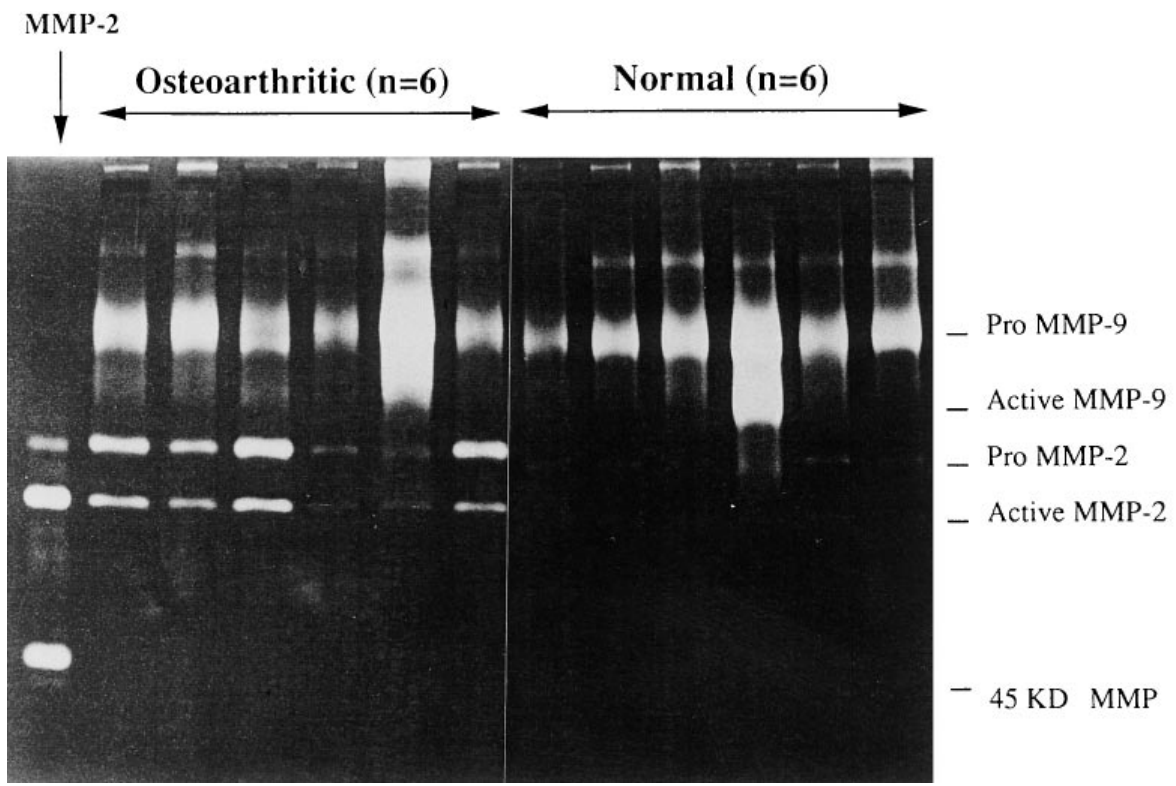

Figure 4. Illustration of a typical gelatin gel zymogram of bone tissue extracts. In this case, the samples are taken from the DC. In four of the six OA tissue specimens, both pro and active gelatinase A (MMP-2) are clearly increased. It is the MMP-2 that is invariably elevated in OA. The MMP-2 standard was of human origin and was purchased from Biogenesis Laboratories. This standard contained both the pro and active enzyme, together with an additional autolysis product which migrated to a position corresponding to a molecular mass of $\sim 45$ $\mathrm{kD}$. Tissue extracts were electrophoresed on the day of their reconstitution in nonreducing buffer. After electrophoresis, gels were developed as for conventional SDSPAGE staining to reveal the proteolytically clarified zones. Confirmation that the observed zones were gelatinases was via loss of the clarified zones when gels were incubated in buffers containing either $5 \mathrm{mM}$ EDTA or $10 \mathrm{mM}$ phenanthroline. In addition, the activities of the enzymes could be adsorbed from samples using gelatin-linked but not casein-linked agarose. Table I summarizes the data obtained for MMP-2 between OA and site-matched control specimens. 
Table I. Tissue Levels of Pro and Active MMP-2 between Normal and $O A$ Femoral Head Cancellous Bone Sites

\begin{tabular}{lcc}
\hline \multirow{2}{*}{ Tissue source } & \multicolumn{2}{c}{ Gelatinase species } \\
\cline { 2 - 3 } & Pro MMP-2 & Active MMP-2 \\
\hline Normal DC & $0.018 \pm 0.006^{*}$ & $0.02 \pm 0.006^{\ddagger}$ \\
OA DC & $0.036 \pm 0.007^{\S}$ & $0.088 \pm 0.016^{\|}$ \\
Normal PC & $0.013 \pm 0.002^{\sharp}$ & $0.021 \pm 0.01^{* *}$ \\
OA PC & $0.148 \pm 0.024$ & $0.41 \pm 0.04$ \\
\hline
\end{tabular}

OA samples, whether from the DC $(* P<0.01)$ or PC $(" \mathbb{P} P<0.0001)$ contained greater levels of pro MMP-2. Similarly, active MMP-2 levels were elevated significantly in both the distal $\left({ }^{\ddagger} P<0.002\right)$ and proximal $\left({ }^{* *} P<0.0001\right)$ sites. Tissue levels for both pro $\left({ }^{\S} P<0.0005\right)$ and active $(\| P<0.0001)$ enzyme exhibited a clear polarization in OA femoral heads, with the proximal zone containing the greater levels. The data represent integrated areas corrected to the area of the standard active MMP-2 \pm SEM.

For the DC, OA tissue contained approximately twice $(P<$ $0.01)$ the pro MMP-2 levels as normal samples (Table I). These differences were even greater for the PC; pro MMP-2 levels from the OA group were $\sim 11.5$-fold higher than normal $(P=$ $0.0001)$ specimens. Similar results were obtained for the active enzyme; in the DC, OA tissue contained $\sim 4.5$-fold more enzyme than normal $(P<0.002)$ samples. As with the proenzyme, the greatest levels for active MMP-2 were associated with the PC. Indeed, the PC from OA samples contained $\sim 19$ times more of the enzyme compared with site-matched normal specimens $(P<0.0001)$. A clear polarization in the levels of pro and active MMP-2 were found only for the OA group, with the greater levels (about four times) located in the PC $(P<$ 0.0001 for the active species and $P<0.0005$ for the zymogen [Table I]).

Gelatinase B (MMP-9) was the major enzyme species found in all the extracts studied, but quantitation was hampered by the presence of material above and below the main zone. These regions are believed to contain differentially glycosylated enzymes together with aggregates and partially degraded forms (24). Clarification was not readily obtained using lower sample loadings.

Insoluble tissue analyses

Collagen content. OA tissue specimens had a greater collagen content than control tissue samples (Fig. 5, and Table II). The

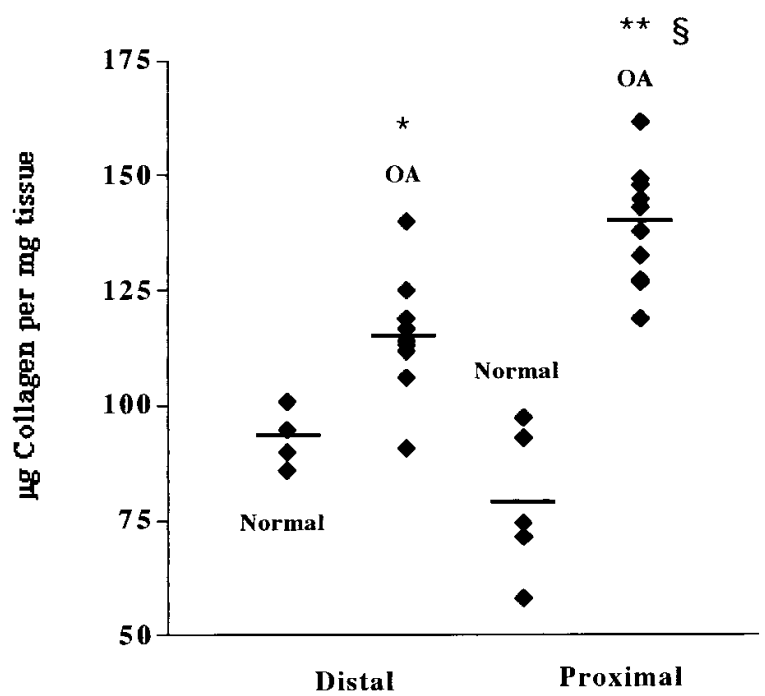

Figure 5. Comparison of the collagen content of bone tissue specimens. The collagen content was based on quantitation of hydroxyproline from tissue hydrolysates. The data are expressed as micrograms of collagen per milligram of the original tissue. OA specimens, whether from the $\mathrm{DC}(* P<0.0005)$ or $\mathrm{PC}(* * P<0.001)$, had greater collagen content compared with site-matched control tissue. The collagen content was also markedly different between distal and proximal zones $\left({ }^{\S} \mathrm{P}<0.0005\right)$ for OA femoral heads.

PC for OA $(140 \pm 3.0 \mu \mathrm{g}$ collagen per mg tissue $)$ contained significantly more collagen $(P<0.001)$ than site-matched normal

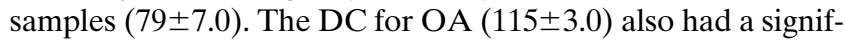
icantly $(P<0.0005)$ greater collagen content compared with the DC of control samples $(93 \pm 2.0)$. Of additional significance is the greater collagen content for the PC of OA compared with the DC $(P<0.0005)$. Normal samples did not differ in their collagen content between the sites examined.

Extent of bone collagen mineralization. Collagen mineral-

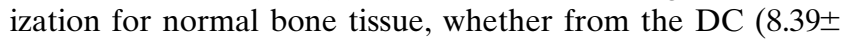
$0.17 \mathrm{mmol}$ calcium per mol collagen) or PC $(8.37 \pm 0.14)$, was greatest $(P<0.001)$ compared with site-matched OA specimens $(7.06 \pm 0.18$ and $6.18 \pm 0.25$, respectively [Fig. 6 , and Table II]). Of additional significance is the difference in mineralization between the proximal and distal sampled sites for OA bone samples, with the former exhibiting a significantly $(P<$ $0.001)$ lower mineralization than the DC.

Collagen cross-linking amino acids. The cross-link content

Table II. Bone Tissue Content of Collagen and the Extent of Collagen Mineralization between OA and Normal Bone Specimens

\begin{tabular}{|c|c|c|c|c|}
\hline \multirow[b]{2}{*}{ Parameter } & \multicolumn{2}{|c|}{ Normal tissue } & \multicolumn{2}{|c|}{ OA tissue } \\
\hline & $\mathrm{PC}$ & $\mathrm{DC}$ & $\mathrm{PC}$ & DC \\
\hline Collagen content ( $\mu$ g collagen/mg tissue) & $79 \pm 7$ & $93 \pm 2$ & $140 \pm 3 *$ & $115 \pm 3^{\ddagger \S}$ \\
\hline Collagen mineralization (mmol calcium/mol collagen) & $8.4 \pm 0.14$ & $8.4 \pm 0.17$ & $6.2 \pm 0.25^{\| 4}$ & $7.2 \pm 0.18 * *$ \\
\hline
\end{tabular}

OA tissue, whether from the PC $(* P<0.001)$ or DC $\left({ }^{*} P<0.0005\right)$ contained significantly more collagen than site-matched control samples. Of additional significance is the greater collagen content $\left({ }^{\circledR} P<0.0005\right)$ for the PC of OA samples versus the DC in these femoral heads. The mineralization of collagen in OA specimens, whether from the PC $(\| P<0.001)$ or DC $(* * P<0.001)$ was significantly lower than that observed for control tissue samples. Furthermore, there was a polarization in the mineralization of OA bone collagen, with the PC having a significantly ( $P<0.001)$ lower calcification than the DC. 


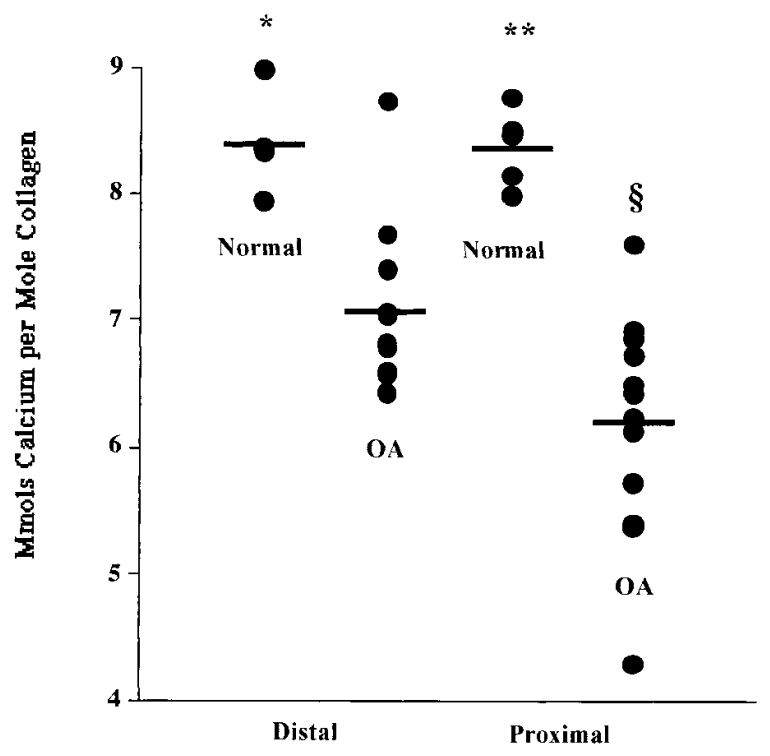

Figure 6. Comparison of the extent of collagen mineralization between OA and normal tissue specimens. Calcium was quantified colorimetrically from a diluted tissue hydrolysate. The degree of tissue mineralization is expressed as millimoles of calcium per mole of collagen. Tissue mineralization, whether from the DC $(*)$ or PC $(* *)$, was greatest $(P<0.001)$ for normal tissue samples. A polarization in collagen mineralization was observed for the OA tissue samples, with the proximal site containing a significantly $\left({ }^{\S} P<0.001\right)$ lower calcium content than the distal region.

of all samples studied was not significantly different (Table III). The highest value for the major immature keto-imine cross-link reduced to DHLNL was within the PC of OA samples but did not reach statistical significance. No changes were observed for the mature pyridinium cross-links between the $\mathrm{OA}$ and controls or between PC and DC of the OA samples.

Bone tissue content of TGF- $\beta$. Samples analyzed for TGF- $\beta$ demonstrated a significantly $(P<0.001)$ greater concentration of this cytokine for the PC of the OA samples, of 123.4 \pm 13.0 ng TGF- $\beta$ per mg tissue extract compared with $32.9 \pm 24.0$ for normal bone extracts. However, it is important to note that the methodology used fails to discriminate between previously ac-

Table III. The Collagen Cross-linking Composition of $O A$ and Normal Cancellous Bone Tissue

\begin{tabular}{lcclll}
\hline & \multicolumn{2}{c}{ Normal tissue } & & \multicolumn{2}{c}{ OA tissue } \\
\cline { 2 - 3 } \cline { 5 - 6 } Cross-link & PC & DC & & PC & \multicolumn{1}{c}{ DC } \\
\hline LHNL & $0.14 \pm 0.04$ & $0.11 \pm 0.02$ & & $0.13 \pm 0.02$ & $0.18 \pm 0.03$ \\
DHLNL & $0.22 \pm 0.05$ & $0.20 \pm 0.02$ & & $0.37 \pm 0.04$ & $0.17 \pm 0.02$ \\
LP & $0.09 \pm 0.01$ & $0.07 \pm 0.01$ & & $0.07 \pm 0.004$ & $0.08 \pm 0.008$ \\
HP & $0.26 \pm 0.04$ & $0.21 \pm 0.02$ & & $0.25 \pm 0.02$ & $0.22 \pm 0.02$ \\
& & & & & \\
\hline
\end{tabular}

In general, the cross-linking is similar for all sites studied. Although not statistically significant, the PC for OA-sampled sites indicates that a greater proportion of the collagen is immature, as demonstrated by the greater concentration of DHLNL. All data are expressed as the mean \pm SEM, where the mean is the moles of cross-link per mole of collagen. $L P$, Lysyl pyridinoline. tive and labile TGF- $\beta$ since all samples underwent activation with the addition of dilute $(40 \mathrm{mM}) \mathrm{HCl}$.

\section{Discussion}

Bone represents the major component of joint tissues, and the metabolism of its collagen framework is pivotal to normal joint function. Our biochemical analyses have demonstrated that OA trabecular bone of the human femoral head is metabolically more active than non-OA controls, and that there is a clear polarization exhibited within the femoral head, the subchondral bone collagen (PC) being the most active.

Elevated type I collagen synthesis as determined by PICP assays, which accurately reflects synthesis of type I collagen (18), is corroborated by increases in AP activity (Fig. 7). Similarly, the predominant immature cross-link (DHLNL) in newly formed bone collagen appeared to be increased almost twofold in the PC compared with controls although it did not reach statistical significance. This is consistent with increased collagen deposition in the subchondral zone (Table II), but the absence of a decrease in the mature pyridinoline cross-links, due to dilution by new collagen, suggests that the rate of deposition is slow, allowing the collagen cross-links to mature. An increased potential for collagen degradation was supported by the increased levels of both pro and active MMP-2; the physiological significance of these findings is emphasized by reports that it is itself capable of cleaving type I and other fibrillar collagens (15) and that some activated forms of MMP-2 are poorly inhibited by tissue inhibitors of MMPs (TIMPs) (25). MMP-2 is activated by an MT1-MMP (16), which is also capable of degrading type I collagen (26) and has been detected in

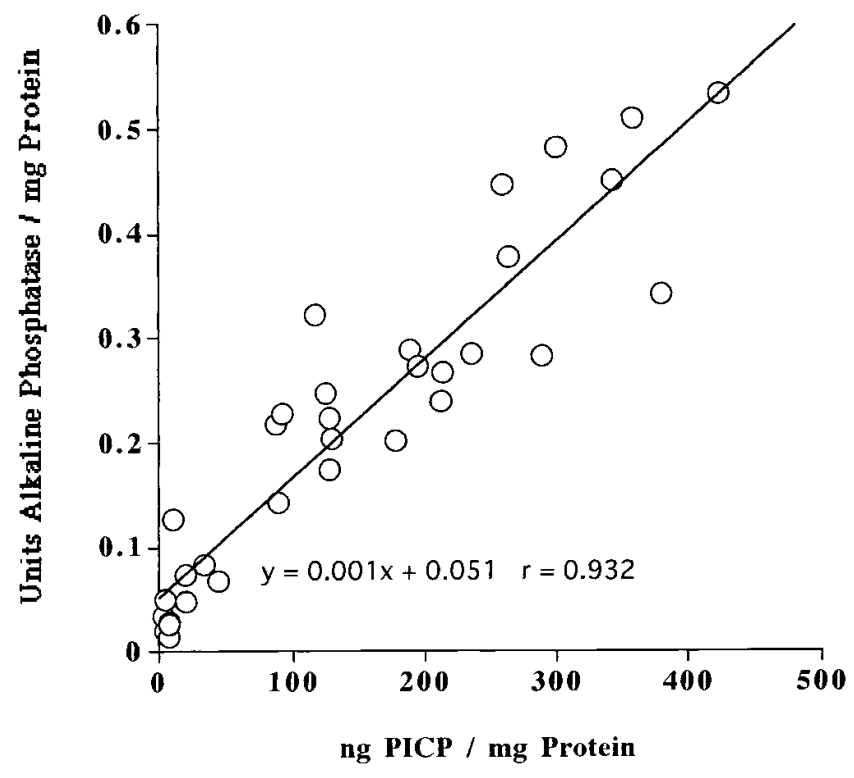

Figure 7. Pearson's correlation between bone tissue PICP and AP. Both the analytes correlate very well $(r=0.93, P<0.001)$. Similar results were obtained with Spearman rank correlation. Although PICP levels reflect the formation of type I collagen, the exact function of AP is unknown but generally thought to be coupled with matrix synthesis. Taken together, PICP and AP from the data depicted provide additional evidence for the association of AP with bone collagen synthesis. 
the cartilage of OA joints (27). Therefore, it is possible, following our identification of greater levels of active MMP-2 in OA cancellous bone, that both MT1-MMP and MMP-2 may be implicated in the increased bone resorption reported for OA subjects (28).

We compared the metabolic activity of the collagen at two sites within the femoral head, the subchondral bone (PC) and tissue near the neck (DC), to determine whether any changes in metabolism were localized. The greatest levels for PICP, $\mathrm{AP}$, and pro and active MMP-2 were in the proximal region, but both PC and DC expressed consistently greater content of these analytes than the controls. The polarization observed for OA tissue was not evident in control femoral heads.

The profound differences in turnover between PC and DC for $\mathrm{OA}$ femoral heads could result in joint deformity, since bone remodeling is a tightly coordinated process fundamental for the shaping and maintenance of tissue morphology. This is highlighted, for example, in the normal modeling process of the rat ulna, where the greater degree of tissue resorption at the medial periosteal surface results in the characteristic curvature of this long bone (29). In hip OA, particularly in the later stages of the disease, one invariably observes femoral head deformation, which may in part be explained by the large differences found in turnover between the two sites studied. Joint deformity in turn could exacerbate the disease process by placing additional stresses and strains on the various tissue compartments to which bone is a particularly sensitive responder (10-12).

Certain growth factors may play a key role in promoting the new bone growth observed. The discovery of TGF- $\beta$ in bone led to the identification of its major role in remodeling and recruitment of osteoblast precursors (30), stimulating collagen synthesis, inhibiting collagenase, and increasing TIMP production, thus leading to a net gain in collagen deposition (31). Our finding of a three- to fourfold increase in TGF- $\beta$ levels in the PC of OA bone versus controls is consistent with the thickening of the subchondral bone through increased matrix deposition. The potential to mineralize OA matrix, as provided by the AP data, is greatest at the PC. However, the content of calcium in relation to the existing collagen (Table II) indicates there is a greater proportion of undermineralized, i.e., immature, recently deposited matrix, which is consistent with the earlier histological findings of a greater proportion of osteoid in OA $(3,4)$.

Type I collagen and AP are both expressed by the mature osteoblasts, which decline in number and function with increasing age. Whether the increased metabolism observed in $\mathrm{OA}$ is a result of increased cellularity and/or activity is unclear. MMP-9, the major gelatinase identified in all extracts and the only species identified in control samples (Fig. 4), has been associated with preosteoblastic cells (32). Roholl and colleagues (33) found that preosteoblasts outweighed the mature phenotype with increasing age. It has been proposed that the greater number of precursor cells is either a consequence of heightened adipocyte generation from a common precursor (34) or simply due to senescence-impaired marrow osteoblast precursor production (35). The ability of TGF- $\beta$ to recruit osteoblast precursors presents a suitable candidate for explaining the increased production of collagen observed in these OA subjects. In addition, the greater levels of MMP-2 in OA tissue samples may support a greater proportion of the mature osteoblast phenotype. A recent in vitro investigation of preosteoblasts
(32) revealed that differentiation to mature cells resulted in the expression of MMP-2.

The thickening of the subchondral bone and its consequent increased stiffness may stimulate cartilage destruction $(6,7)$, or the latter could possibly stimulate a thickening of bone as a protective measure. Alternatively, both tissues may be affected simultaneously as a result of some unknown biochemical interactions between the two tissues. Certainly, the demonstration of high levels of metabolic activity in the subchondral zone opens up the question of the role of bone collagen in OA. Whether thickening of the subchondral bone precedes cartilage fibrillation is an interesting question and a subject of some debate (36).

The increased metabolism of bone and the deposition of increased collagen may be features of other bones in OA subjects. Dequeker et al. (37) have reported increased bone density in OA, which they suggest protects against hip fracture. Other workers (38) confirmed increased bone density in OA but could not confirm the absence of hip fractures. The contrasting status of bone density between OA and osteoporosis (39) is evident in the virtual absence of OA changes in the fractured hips of osteoporotic subjects. The general but still controversial opinion is that increased bone mass increases the risk of developing OA (40-42). However, a high bone density is unlikely to be the sole prerequisite for a predisposition to OA, since African American men and women have a greater bone mass than Caucasians (43-45) yet are no more prone to the disease (1). However, African Americans, like Caucasians, will lose bone after the fourth decade. An explanation for the greater risk of developing OA with increased bone mass may be that it is due to a net accumulation of bone, since subjects who reach adulthood with osteopetrosis are strongly associated with the development of OA (46). Clearly, the type of investigation covered in this study if applied to other bones could provide additional valuable data to resolve this question.

What is evident from this study is that OA cancellous bone is metabolically very active compared with normal tissue, in particular the proximal sampled sites. Such differences in turnover might result in altered joint morphology, which in turn might exacerbate the disease process. OA is a cruelly disabling and painful disease, which is assuming greater significance especially in more developed countries. It is anticipated that the results presented here will stimulate a greater effort toward studying bone metabolism and its role in the pathogenesis of OA.

\section{Acknowledgments}

The authors wish to thank Dr. Gordon Paul for his assistance with statistical analyses, and the Photographic Unit, University of Bristol, Langford, for the preparation of several figures used in this report. We would also like to thank Mr. Trevor Sims of the Collagen Research Group, University of Bristol, Langford, for analyzing tissue samples for the collagen cross-linking amino acids. In addition, we thank the Biochemistry Unit, University of Bristol, Langford, for analysis of samples for AP, total protein, and calcium.

J.P. Mansell is indebted to the Arthritis and Rheumatism Council for their funding.

\section{References}

1. Kirwan, J.R., and A.J. Silman. 1987. Epidemiological, sociological and environmental aspects of rheumatoid arthritis and osteoarthrosis. Bailliere's 
Clin. Rheumatol. 1:467-489.

2. Plewes, A. 1940. Osteo-arthritis of the hip. Br. J. Surg. 27:682.

3. Batra, H.C., and J. Charnley. 1969. Existence and incidence of osteoid in osteoarthritic femoral heads. J. Bone Jt. Surg. Br. Vol. 51:366-371.

4. Reinmann, I., H.J. Mankin, and C. Trahan. 1977. Quantitative histologic analyses of articular cartilage and subchondral bone from osteoarthritic and normal human hips. Acta Orthop. Scand. 48:63-73.

5. Jeffery, A.K. 1973. Osteogenesis in the osteoarthritic femoral head. J. Bone Jt. Surg. Br. Vol. 55:262-272.

6. Radin, E.L., P.J. Abernethy, P.M. Townsend, and R.M. Rose. 1978. The role of bone changes in the degeneration of articular cartilage in osteoarthritis. Acta Orthop. Belg. 44:55-63.

7. Radin, E.L., and R.M. Rose. 1986. Role of subchondral bone in the initiation and progression of cartilage damage. Clin. Orthop. Relat. Res. 213:34-40.

8. Dieppe, P., J. Cushnaghan, P. Young, and J. Kirwan. 1993. Prediction of the progression of joint space narrowing in osteoarthritis of the knee by bone scintigraphy. Ann. Rheum. Dis. 52:557-563.

9. Linsenmeyer, T.F., E. Gibney, F. Igoe, M.K. Gordon, L.I. Fessler, and D.E. Birk. 1993. Type V collagen: molecular structure and fibrillar organization of the chicken $\alpha \mathrm{I}(\mathrm{V}) \mathrm{NH}_{2}$-terminal domain, a putative regulator of corneal fibrillogenesis. J. Cell Biol. 121:1181-1189.

10. Rodan, G.A. 1991. Mechanical loading, estrogen deficiency, and the coupling of bone formation to bone resorption. J. Bone Miner. Res. 6:527-529.

11. Rubin, C.T., and L.E. Lanyon. 1985. Regulation of bone mass by mechanical strain magnitude. Calcif. Tissue Int. 37:411-417.

12. Pead, M.J., T.M. Skerry, and L.E. Lanyon. 1988. Direct transformation from quiescence to bone formation in the adult periosteum following a single brief period of bone loading. J. Bone Miner. Res. 3:647-656.

13. Mansell, J.P., J.F. Tarlton, and A.J. Bailey. 1997. Biochemical evidence for altered subchondral bone collagen metabolism in osteoarthritis of the hip.

Br. J. Rheumatol. 36:16-19.

14. Singer, F.R., and G.D. Roodman. 1996. Paget's disease of bone. In Principles of Bone Biology. J.P. Bilezikian, L.G. Raisz, and G.A. Rodan, editors. Academic Press, Inc., San Diego. 969-977.

15. Aimes, R.T., and J.P. Quigley. 1995. Matrix metalloproteinase-2 is an interstitial collagenase. Inhibitor-free enzyme catalyzes the cleavage of collagen fibrils and soluble native type I collagen generating the specific 3/4- and 1/4length fragments. J. Biol. Chem. 270:5872-5876.

16. Sato, H., T. Takahisha, Y. Okada, J. Cao, A. Shinagawa, E. Yamamoto, and M. Selki. 1994. A matrix metalloproteinase expressed on the surface of invasive tumour cells. Nature. 370:61-65.

17. Mansell, J.P., J.F. Tarlton, and A.J. Bailey. 1997. Expression of gelatinases within the trabecular bone compartment of ovariectomized and parathyroidectomized adult female rats. Bone (NY). 20:533-538.

18. Risteli, J., and L. Risteli. 1995. Analysing connective tissue metabolites in human serum. Biochemical, physiological and methodological aspects. $J$. Hepatol. 22(Suppl. 2):77-81.

19. Nefussi, J.R., M. Pouchelet, P. Collin, J.M. Sautier, G. Develay, and N. Forest. 1989. Microcinematographic and autoradiographic kinetic studies of bone cell differentiation in vitro: matrix formation and mineralization. Bone (NY). 10:345-352

20. Laemmli, U.K. 1970. Cleavage of structural proteins during assembly of head bacteriophage T4. Nature. 227:680-685.

21. Sims, T.J., and A.J. Bailey. 1992. Quantitative analysis of collagen and elastin cross-links using a single-column system. J. Chromatogr. 582:49-55.

22. Bannister, D.W., and A.B. Burns. 1970. Adaptation of the Bergman and Loxley technique for hydroxyproline determination to the autoanalyzer and its use in determining plasma hydroxyproline in the domestic fowl. Analyst. 95: $596-600$

23. Dequeker, J., S. Mohan, R.D. Finkelman, J. Aerssens, and D.J. Baylink. 1993. Generalized osteoarthritis associated with increased insulin-like growth factor types I and II and transforming growth factor beta in cortical bone from the iliac crest. Possible mechanism of increased bone density and protection against osteoporosis. Arthritis Rheum. 36:1702-1708.

24. Delaisse, J.M., Y. Eeckhout, L. Neff, C. Francois-Gillet, P. Henriet, Y. $\mathrm{Su}, \mathrm{G}$. Vaes, and R. Baron. 1993. (Pro)collagenase (matrix metalloproteinase-1) is present in rodent osteoclasts and in the underlying bone-resorbing compartment. J. Cell Sci. 106:1071-1082.

25. Bullen, E.C., M.T. Longaker, D.L. Updike, R. Benton, D. Ladin, Z.
Hou, and E.W. Howard. 1995. Tissue inhibitor of metalloproteinases-1 is decreased and activated gelatinases are increased in chronic wounds. J. Investig. Dermatol. 104:236-240.

26. Ohuchi, E., K. Imai, Y. Fujii, H. Sato, M. Seiki, and Y. Okada. 1997. Membrane type I matrix metalloproteinase digests interstitial collagens and other extracellular matrix macromolecules. J. Biol. Chem. 272:2466-2451.

27. Butter, F.H., S. Chubenskaya, D. Margerie, K. Huch, J. Flechtenmacher, A.A. Cole, K.E. Kuettner, and E. Bartnik. 1997. Expression of membrane type I matrix metalloproteinase in human articular cartilage. Arthritis Rheum. 40:704-709.

28. Seibel, M.J., A. Duncan, and S.P. Robins. 1989. Urinary hydroxy-pyridinium crosslinks provide indices of cartilage and bone involvement in arthritic diseases. J. Rheumatol. 16:964-970.

29. Hillam, R.A., and T.M. Skerry. 1995. Inhibition of bone resorption and stimulation of formation by mechanical loading of the modelling rat ulna in vivo. J. Bone Miner. Res. 10:683-689.

30. Pfeilschifter, J., O. Wolf, A. Nautmann, H.W. Minne, G.R. Mundy, and R. Zeigler. 1990. Chemotactic response of osteoblast-like cells to TGFß. $J$. Bone Miner. Res. 5:825-830.

31. Bonewald, L.F. 1996. Transforming growth factor- $\beta$. In Principles of Bone Biology. J.P. Bilezikian, L.G. Raisz, and G.A. Rodan, editors. Academic Press, Inc., San Diego. 969-977.

32. Rifas, L., A. Fausto, M.J. Scott, L.V. Avioli, and H.G. Welgus. 1994. Expression of metalloproteinases and tissue inhibitors of metalloproteinases in human osteoblast-like cells: differentiation is associated with repression of metalloproteinase biosynthesis. Endocrinology. 134:213-221.

33. Roholl, P.J.M., E. Blauw, C. Zurcher, J.A.M.A. Dormans, and H.M. Theons. 1994. Evidence for a diminished maturation of preosteoblasts into osteoblasts during aging in rats: an ultrastructural analysis. J. Bone Miner. Res. 9: 355-366.

34. Beresford, J.N., J.H. Bennett, C. Devlin, P.S. Leboy, and M.E. Owen. 1992. Evidence for an inverse relationship between the differentiation of adipocytic and osteogenic cells in rat marrow stromal cell cultures. J. Cell Sci. 102: 341-351.

35. Manolagas, S.C., and R.L. Jilka. 1995. Bone marrow, cytokines, and bone remodelling. Emerging insights into the pathophysiology of osteoporosis N. Engl. J. Med. 332:305-311.

36. Bailey, A.J., and J.P. Mansell. 1997. Do subchondral bone changes exacerbate or precede articular cartilage destruction in osteoarthritis of the elderly? Gerontology. 43:296-304.

37. Dequeker, J., L. Mokassa, and J. Aerssens. 1995. Bone density and osteoarthritis. J. Rheumatol. Suppl. 43:98-100.

38. Jones, G., T. Nguyen, P.N. Sambrook, S.R. Lord, P.J. Kelly, and J.A Eisman. 1995. Osteoarthritis, bone density, postural stability, and osteoporotic fractures: a population based study. J. Rheumatol. 22:921-925.

39. Dequeker, J., and O. Johnell. 1993. Osteoarthritis protects against femoral neck fracture: the MEDOS study experience. Bone (NY). 14:S51-S56.

40. Lane, N.E., and J.C. Nevitt. 1994. Osteoarthritis and bone mass. J. Rheumatol. 21:1393-1396.

41. Sowers, M.F., M. Hochberg, J.P. Crabbe, A. Muhich, and M. Crutchfield. 1996. Osteoarthritis of the hand and knee in premenopausal women. Am. J. Epidemiol. 143:38-47.

42. Marcelli, C., F. Favier, P.O. Kotzki, V. Ferrazzi, M.C. Picot, and L. Simon. 1995. The relationship between osteoarthritis of the hands, bone mineral density, and osteoporotic fractures in elderly women. Osteoporos. Int. 5:382-388.

43. Nelson, D.A., G. Jacobsen, D.A. Barondess, and A.M. Parfitt. 1995 Ethnic differences in regional bone density, hip axis, length, and lifestyle variables among healthy black and white men. J. Bone Miner. Res. 10:782-787.

44. Bell, N.H., L. Gordon, J. Stevens, and J.R. Shary. 1995. Demonstration that bone mineral density of the lumbar spine, trochanter, and femoral neck is higher in black than in white young men. Calcif. Tissue Int. 56:11-13.

45. Kleerekoper, M., D.A. Nelson, E.L. Peterson, M.J. Flynn, A.S. Pawluszka, G. Jacobsen, and P. Wilson. 1994. Reference data for bone mass, calciotrophic hormones, and biochemical markers of bone remodeling in older (55-75) postmenopausal white and black women. J. Bone Miner. Res. 9:12671276.

46. Milgram, J.W., and M. Jasty. 1982. Osteopetrosis. A morphological study of twenty-one cases. J. Bone Jt. Surg. Am. Vol. 64:912-929. 